\title{
Pólya urns: Probabilistic analysis and statistical questions
}

\author{
Hosam M. Mahmoud \\ Department of Statistics \\ The George Washington University \\ Washington, DC 20052, U.S.A.
}

Pólya urns have become an indispensable mathematical and modeling tool for the understanding of many areas, such as engineering, economics, epidemiology, informatics (only to name a few of the plethora of applications). In this talk we present many fundamental results about Pólya urns, with a focus on the more recent area of multiple ball drawing. Potential applications will be mentioned. We leave the audience with a few statistical questions about Pólya urns. 